\title{
The potential role of Colchicine in preventing coronary vascular disease in childhood-onset lupus: a new view on an old drug
}

\author{
Dori Abel ${ }^{1 *}$, Stacy P. Ardoin ${ }^{2,3}$ and Mark Gorelik ${ }^{4}$
}

\begin{abstract}
Background: Patients with systemic lupus erythematous have a significantly increased risk of cardiovascular disease, which is not fully explained by traditional cardiovascular disease risk factors. Despite increasing life expectancy in patients with systemic lupus erythematous, mortality due to cardiovascular disease, the major cause of death in these patients, has not changed. Children with lupus suffer from more aggressive disease compared to their adult counterparts, and there is a growing concern for their increased risk of cardiovascular disease as they age.
\end{abstract}

Body:: There is an unmet need for therapies to address the increased risk of cardiovascular disease in childhoodonset lupus. Colchicine has many anti-inflammatory and cardiovascular protective properties, including inhibition of IL-1 $\beta$ and IL-18 activity, key proinflammatory cytokines that are predictive of future adverse cardiovascular events. In the Colchicine Cardiovascular Outcomes Trial (COLCOT), colchicine was recently found to have significant benefit with minimal risk in adults with previous myocardial infarction for prevention of secondary vascular disease. While adult studies are promising, no studies have been conducted in pediatric patients to investigate colchicine's potential for cardiovascular protection in children and adolescents with lupus.

Conclusions: Studies investigating colchicine's potential role for cardiovascular protection are needed in pediatric patients with systemic lupus erythematous.

Keywords: Systemic lupus erythematous, Cardiovascular Disease, Colchicine, Atherosclerosis, Prevention

\section{Background}

Due to advances in diagnosis and management of systemic lupus erythematous (SLE), patients are living longer. There is now increased focus on detection and prevention of long-term complications and downstream associated comorbidities in SLE, including atherosclerosis [1]. While all-cause mortality in patients with SLE

\footnotetext{
* Correspondence: dna9003@nyp.org

'Department of Pediatrics, NewYork-Presbyterian Hospital, Columbia University Irving Medical Center, 630 W. 168th Street, New York, NY 10032-3702, USA

Full list of author information is available at the end of the article
}

has declined since 1975, mortality due to cardiovascular disease (CVD), the major cause of death in these patients, has remained stagnant [2]. One hypothesis for this discordance is that increased life expectancy in patients with SLE has led to more patients ultimately suffering from CVD [3]. Although atherosclerosis worsens with age, increased life expectancy does not fully explain this unchanged cardiovascular mortality [4]. Women under age 45 with SLE are over 50 times more likely to suffer a myocardial infarction (MI) than women of similar age in the Framingham Offspring Study [4], 
suggesting other factors than age contribute to CVD mortality in lupus patients. CVD is also significantly more common in young premenopausal women with SLE compared to controls [4].

Compared to their adult counterparts, children with SLE have more aggressive disease, a lower life expectancy, longer disease burden, higher incidence of lupus nephritis and neurological manifestations, and there is growing concern for their increased risk of CVD as they age [5-8]. Indeed, adults with history of childhood-onset SLE have a standardized mortality ratio of 2.5 , with CVD the most common reported cause of death, and they experience these complications at an early age, with average age of first MI as early as $32[7,9]$. Both traditional Framingham CVD risk factors and SLE-specific risk factors, primarily related to the immune-mediated pathogenesis of the disease, make SLE itself a clear, independent risk factor for CVD [10].

Despite recommendations to screen patients with SLE for CVD risk factors and to encourage healthy diets and exercise [11, 12], providers inconsistently screen [13]. Even when risk factors are identified, such as hypertension, diabetes, hyperlipidemia, and obesity, traditional interventions including behavioral modifications and medical management are insufficient to prevent CVD [13], which is unsurprising given the presence of nonclassical, SLE-specific risk factors. Research and new therapies are needed to prevent premature atherosclerosis in pediatric patients with SLE.

Colchicine, known for its use in gout, Familial Mediterranean Fever (FMF), and other IL- $1 \beta$ mediated syndromes, has many anti-inflammatory and cardiovascular protective properties [14]. In particular, underscoring its utility in fever syndromes, colchicine has effects in inhibiting IL-1 $\beta$ activity via inhibition of the NALP3 inflammasome [14]. The Colchicine Cardiovascular Outcomes Trial (COLCOT) recently demonstrated that colchicine therapy provided significant benefit in prevention of secondary CVD for adults with previous MI [15], suggesting potential promise for CVD protection in other populations. Studies investigating colchicine's potential role for cardiovascular protection are needed in children and adolescent patients with SLE. This article will review the current knowledge and background in therapy regarding cardiac atherosclerosis and coronary artery disease in childhood-onset and adult lupus, and will conclude by proposing investigation on the use of colchicine for prevention of CVD in young patients with SLE.

\section{Main text}

\section{Role of inflammation in Atherosclerosis and CVD}

All three components of the immune system - innate, cellular, and humoral immunity - are involved in the development of atherosclerosis. Differentiated macrophages upregulate receptors implicated in the innate immune response, including oxLDL and toll-like receptors [16]. Activation of these receptors results in the development of foam cells and the release of cytokines, vasoactive molecules, proteases, and oxidative species that contribute to plaque destabilization [17]. T-cells, in particular Th1 cells, also promote atherogenesis through their release of proinflammatory cytokines, including tumor necrosis factor (TNF), interferon- $\gamma$, plateletderived growth factor, interleukin (IL)-1 $\beta$, IL-2, IL-6, IL8 , and IL-12 [16, 18]. These cytokines and growth factors foster smooth muscle migration and proliferation, expanding the atherosclerotic plaque [19]. Autoantigens and autoantibodies, hallmarks of the humoral immune response, further contribute to atherosclerosis. Elevated levels of autoantibodies, including anti-oxLDL antibodies, anti-phospholipid antibodies, anti-cardiolipin antibodies, anti- $\beta 2 \mathrm{GP} 1$ antibodies, and anti-HSP antibodies, which contribute to endothelial damage and interrupt normal anti-atherosclerotic defenses, have been associated with atherosclerosis and thrombosis, and autoantigens within vessel walls and cholesterol molecules also play a role in inflammatory processes underlying atherogenesis [18].

Emerging evidence suggests that, of all these immunologic factors, IL- $1 \beta$ plays a primary role in the development of CVD [20-22]. Specifically, IL-1 $\beta$ promotes monocyte and leukocyte adhesion to vascular endothelium and development of atherothrombotic plaques [2022]. In animal trials, blockade of IL-1 $\beta$ decreased atherosclerotic lesions and intimal thickening [23, 24]. Prior to the COLCOT trial, the Canakinumab Anti-inflammatory Thrombosis Outcome Study (CANTOS) took a definitive clinical step in highlighting the role of IL-1 $\beta$ by demonstrating that canakinumab therapy showed benefit in preventing secondary vascular events in adults after MI [25]. While complicated by a small but significant signal of fatal infection, conceptually this trial firmly established the role of IL-1 $\beta$ as a major driver of CVD [25].

\section{Increased risk of CVD in patients with SLE}

Atherosclerosis is known to begin in adolescence even in the absence of SLE [26], yet patients with childhoodonset lupus experience accelerated atherosclerosis and early CVD $[1,2,8]$. Dyslipidemia is present in $63-85 \%$ of children and adolescents with lupus even at initial diagnosis $[27,28]$, compared to approximately $20 \%$ of the general pediatric population [29]. Despite premature atherosclerosis, clinical CVD manifested by MI and angina pectoris in pediatric patients with lupus is rare [4, 30, 31]. However, children with lupus do experience other acute cardiac diagnoses more commonly, with pericarditis being most common at about $10 \%$, followed 
closely by valvular insufficiency [32]. Myocarditis and endocarditis are less commonly reported in children [32]. It is possible that this early acute pericardial involvement and valvular insufficiency contribute to longterm CVD as the initial cardiac insults in some patients [33, 34].

As recognition of the high cardiovascular morbidity and mortality in young patients with SLE has grown, many studies have focused on the identification and understanding of factors associated with this increased risk. Analyses from the Atherosclerosis Prevention in Pediatric Lupus Erythematosus (APPLE) trial, during which patients underwent baseline measurements of carotid IMT (CIMT), demonstrate that increased CIMT is associated with both traditional and nontraditional CVD risk factors, including increased age, longer disease duration, minority status, higher body mass index, male sex, increased creatinine clearance, higher lipoprotein(a) level, proteinuria, azathioprine treatment, and prednisone dose [35].

\section{latrogenic risk factors}

While sometimes overlooked, some of the medications used to reduce inflammation in SLE increase CVD risk by potentiating classical risk factors. Corticosteroids have many known adverse effects, including impaired glucose tolerance, hypertension, and obesity, which amplify these known traditional CVD risk factors. Azathioprine has also been associated with higher CIMT measurements in pediatric patients with SLE and increased rates of clinical CVD [35, 36], unlike the immunosuppressant agents mycophenolate mofetil and cyclophosphamide, which have not demonstrated significant impact on lipid profiles or CIMT [35, 37]. Conversely, hydroxychloroquine may be cardioprotective, by improving lipid profiles [38] and vessel elasticity [39]. This positive medication effect, however, is not enough to outweigh the many other factors that contribute to increased CVD risk in patients with lupus.

\section{Statins and the APPLE trial}

Statins, which exert their effects through inhibition of 3hydroxy-3-methyl-glutarylcoenzyme A (HMG-coA) reductase, the rate-limiting step in cholesterol synthesis, are first-line medications to treat hyperlipidemia and to prevent cardiovascular events in adults at risk of CVD. Statins also affect pathways related to inflammation and have many vasculoprotective effects [40], offering hope that they may prevent atherosclerosis in SLE, but studies in both adults and children with SLE have failed to show significant benefit $[41,42]$. The Lupus Atherosclerosis Prevention Study (LAPS) of adult patients with SLE without clinical CVD found no difference in either the primary outcome (coronary artery calcium) or secondary outcomes (CIMT and carotid plaque) between the group treated with atorvastatin compared to placebo [41]. There was also no reduction in high sensitivity Creactive protein (hsCRP) levels or any markers of endothelial activation, and patients who received atorvastatin experienced more liver toxicities than the control group.

Given that young patients have fewer comorbidities and traditional cardiovascular risk factors than adults and thus represent a unique population, the APPLE trial was designed to assess the use of statins in children and adolescents with SLE [42]. This multicenter, randomized, double-blind, placebo-controlled trial investigated the 3-year efficacy and safety of atorvastatin in preventing subclinical atherosclerosis progression in childhoodonset SLE. Although there was a general positive effect of treatment on slowing common CIMT in patients receiving atorvastatin, the results of this robust trial were not statistically significant, and thus routine use of statins in pediatric patients with lupus could not be supported. Unlike the LAPS in which atorvastatin was associated with liver toxicities, there was no concerning safety signal in children. Both the placebo and treatment groups experienced significant progression in CIMT over the 3-year period, and at a faster rate than that expected in the general population, supporting the understanding that subclinical atherosclerosis in patients with SLE begins in childhood. This highlights the critical need for routine screening and management of CVD risk factors in pediatric rheumatology practice.

A secondary analysis of the APPLE study was conducted to evaluate whether certain subgroups, categorized by duration of disease, pubertal status, and variables linked to cardiovascular risk and CIMT, would benefit from statin therapy [43]. In this analysis, postpubertal status and higher hsCRP levels were associated with lower CIMT progression in patients treated with atorvastatin, and this effect was greatest in the combined post-pubertal and high hsCRP group. Although secondary analyses must be interpreted with caution, these findings suggest that a more personalized approach should be considered for CVD risk reduction in SLE, as certain high-risk cohorts may benefit from more aggressive treatment strategies, including statin therapy and potentially other cardiovascular-protective interventions.

\section{Colchicine: mechanism, role in CVD, and potential use in SLE}

Colchicine, an alkaloid initially extracted from the $a u$ tumn crocus plant, is an anti-inflammatory medication used for over 2000 years to treat gout [44]. While its pharmacologic mechanisms are not completely understood, colchicine's primary mechanism is via its binding to tubulins, forming a complex that interferes with polymerization of microtubules, a key component of the 
cytoskeleton [14, 45]. Colchicine's impact on microtubules results in multiple downstream effects on mitotic activity, malignancy, and inflammation. Its antiinflammatory actions include interference with neutrophil chemotaxis, adhesion, and mobilization through microtubule polymerization [46, 47], and suppression of superoxide production from neutrophils [48]. Importantly, colchicine also inhibits NALP3 (also known as cryopyrin) inflammasomes, thereby inhibiting caspase- 1 activation and subsequently preventing nuclear factor $\mathrm{kB}$ activation and the production of active IL- $1 \beta$ and IL-18 $[49,50]$. IL-1 $\beta$ is involved in monocyte and leukocyte adhesion to vascular endothelial cells, growth of vascular smooth muscle cells, and coagulation induction, and it stimulates the downstream IL-6 receptor signaling pathway, which promotes expression of fibrinogen and plasminogen activator factor, two important thrombotic mediators [51-53]. Both IL-1 $\beta$ and IL-18 (a member of the IL-1 family) are key proinflammatory cytokines and predictive of future adverse cardiovascular events, offering potential for colchicine as a cardioprotective therapeutic intervention [51].

Colchicine also has anti-fibrotic and cardiovascular benefits. It has shown potential in preventing and even reversing both amyloidosis and endothelial dysfunction in patients with FMF [54], and it reduces intimal hyperplasia by down-regulating leukocyte vascular endothelial growth factor expression after angioplasty in dogs [55]. In rat pulmonary arterial hypertension models, it reduced smooth muscle cell proliferation, increased cell apoptosis, and reduced expression of TNF- $\alpha$ and NF- $\mathrm{kB}$ both alone and when combined with a vasodilator [56], and it demonstrated synergy with atorvastatin in improving endothelial function and reducing inflammation in rats with hyperlipidemia [57].

Colchicine is currently used to treat various inflammatory conditions, including gout, FMF, Behcet's disease, and recurrent pericarditis. Pericarditis associated with lupus has traditionally been treated with corticosteroids, although colchicine demonstrated effectiveness and was well tolerated in a series of 10 cases of lupus with pericarditis [58]. Colchicine has also exhibited long-term safety in studies of children with FMF with minimal adverse effects. A minority of patients experience transient transaminitis and transient, dose-dependent diarrhea, which resolves with split doses and/or dose reduction $[59,60]$. Multiple long-term studies have supported colchicine's tolerability in children of all ages with FMF; in two studies, one with 153 patients and another with 350 patients, colchicine was not discontinued in any of the patients over a period of 4 years and 6-13 years, respectively $[59,61]$. Because colchicine is metabolized in the liver via a cytochrome P450 3A4-dependent pathway [62], caution should be taken when co-administering colchicine with other medications, notably cytochrome P450 3A4 inhibitors [60, 62].

Given its anti-inflammatory properties and tolerable side effect profile, colchicine has recently been studied in the context of cardiovascular protection. In the LowDose Colchicine (LoDoCo) trial, colchicine reduced cardiovascular events in patients with stable coronary disease, although this initial trial was small and unblinded [63]. Building on the LoDoCo trial, the large, randomized, double-blind, placebo-controlled COLCOT trial was conducted to evaluate the effects of colchicine on cardiovascular events as well as its long-term safety profile in patients who had recently experienced a MI [15]. Among these patients, those who received colchicine at a dose of $0.5 \mathrm{mg}$ daily had a significantly lower risk of ischemic cardiovascular events (predominantly strokes and urgent hospitalizations for angina leading to coronary revascularization) compared to the placebo group. The most common side effects experienced by both groups were gastrointestinal, although there was no statistical difference in any gastrointestinal-related adverse event, including diarrhea. Pneumonia, however, did show statistical significance as a serious adverse event, although this event was uncommon overall, seen in $0.9 \%$ of patients in the colchicine group and $0.4 \%$ of those in the placebo group.

\section{Potential approach for future study}

The APPLE trial laid invaluable groundwork for understanding how to approach future study of CVD in pediatric patients with SLE. Lessons from this trial suggest that robust methods of detecting early and subclinical vascular inflammation are needed to ensure a reliable and definable end point for a clinical study. A recent study in healthy adult patients suggests that CVD progresses rapidly in the fifth decade of life [64]. This study somewhat alters our paradigm of CVD as a slowly progressive, lifelong process, but it also may imply that factors which arise in healthy individuals to promote CVD during this fifth decade are accelerated by SLE, and that it is possible to detect these factors by imaging and molecular methods. One potential approach for a future study is to define these factors with more precision through collaboration with the wider scientific community, and then utilize them as markers for accelerated CVD in our patient population. At the same time, preliminary, well-powered studies of the effect of colchicine in childhood-onset lupus, using a broad array of currently known markers of subclinical CVD, are also needed and will add to our understanding. Following these studies, a broader trial of colchicine in pediatric patients with SLE for the prevention of CVD, with identifiable and measurable endpoints that clearly allow us to measure benefit, should be a real possibility. Since 
morbidity and mortality from accelerated atherosclerosis is uncommonly seen in the pediatric population with SLE, these studies in pediatric patients should extend into adulthood.

Study design in the form of a multi-center, randomized, double-blind, placebo-controlled clinical trial would most robustly capture potential benefits. Drawing from lessons of previous trials, selecting post-pubertal patients and including young adults may allow investigators to capture patients most at risk for CVD progression and thus most likely to benefit from an intervention. These participants, followed over both the medium- and longterm, would be assessed with carotid IMT, investigational serum markers of endothelial dysfunction such as specific circulating cytokines [65] including IL-18 and IL-6, or endothelial function mediators such as endothelial nitric oxide synthase [66]. Longer-term follow up could measure coronary artery calcium scores and the emergence of major adverse cardiac events in study groups.

\section{Conclusions}

Given colchicine's clear anti-inflammatory benefits and effectiveness in secondary prevention of CVD in adults, this drug offers hope for prevention of CVD in children and adolescents with SLE, a known inflammatory condition with long-term effects on the heart and vasculature. To our knowledge, no studies have been conducted investigating colchicine's potential for cardiovascular protection in pediatric patients with SLE. The need for prevention of CVD in our patients is nothing less than paramount. As pediatric rheumatologists, it is our duty to allow our patients to enjoy full and complete lives despite their disease, both as children and beyond.

\section{Abbreviations \\ SLE: Systemic lupus erythematous; CVD: Cardiovascular disease; MI: Myocardial infarction; FMF: Familial Mediterranean Fever; COLCOT: Colchicine Cardiovascular Outcomes Trial; TNF: Tumor necrosis factor; IL: Interleukin; CANTOS: Canakinumab Anti-inflammatory Thrombosis Out- come Study; APPLE: Atherosclerosis Prevention in Pediatric Lupus Erythematous; CIMT: Carotid IMT; LAPS: Lupus Atherosclerosis Prevention Study; hsCRP: High sensitivity C-reactive protein; LoDoCo: Low-Dose Colchicine}

\section{Acknowledgements}

not applicable.

\section{Authors' contributions}

DA appraised the literature and wrote the manuscript. SA revised the manuscript and contributed to the conception of the manuscript. MG revised the manuscript, contributed to the conception of the manuscript, and coordinated the authors' work. All authors critically reviewed the manuscript for relevant intellectual content. All authors approved this submitted version of the manuscript. All authors have agreed both to be personally accountable for the author's own contributions and to ensure that questions related to the accuracy or integrity of any part of the work, even ones in which the author was not personally involved, are appropriately investigated, resolved, and the resolution documented in the literature.
Funding

none.

Availability of data and materials

not applicable.

Ethics approval and consent to participate

not applicable.

Consent for publication

not applicable.

\section{Competing interests}

The authors declare that they have no competing interests.

\section{Author details}

${ }^{1}$ Department of Pediatrics, NewYork-Presbyterian Hospital, Columbia University Irving Medical Center, 630 W. 168th Street, New York, NY 10032-3702, USA. ${ }^{2}$ Department of Medicine, Division of Rheumatology and Immunology, The Ohio State University, 370 W. 9th Ave, Columbus, $\mathrm{OH}$ 43210, USA. ${ }^{3}$ Department of Rheumatology, Nationwide Children's Hospital, 700 Children's Dr, Columbus, OH 43205, USA. ${ }^{4}$ Department of Pediatrics, Division of Allergy, Immunology, and Rheumatology, Columbia University Irving Medical Center, 630 W. 168th St, New York, NY 10032-3702, USA.

Received: 14 August 2020 Accepted: 8 February 2021

Published online: 16 February 2021

\section{References}

1. Sandborg C, Ardoin SP, Schanberg L. Therapy Insight: cardiovascular disease in pediatric systemic lupus erythematosus. Nat Clin Pract Rheumatol. 2008; 4(5):258-65.

2. Bjornadal L, Yin L, Granath F, Klareskog L, Ekbom A. Cardiovascular disease a hazard despite improved prognosis in patients with systemic lupus erythematosus: results from a Swedish population based study 1964-95. J Rheumatol. 2004:31(4):713-9.

3. Kiani AN, Post WS, Magder LS, Petri M. Predictors of progression in atherosclerosis over 2 years in systemic lupus erythematosus. Rheumatology. 2011;50(11):2071-9.

4. Manzi S, Meilahn EN, Rairie JE, Conte CG, Medsger TA Jr, Jansen-McWilliams $L$, et al. Age-specific incidence rates of myocardial infarction and angina in women with systemic lupus erythematosus: comparison with the Framingham Study. Am J Epidemiol. 1997;145(5):408-15.

5. Tucker LB, Uribe AG, Fernandez M, Vila LM, McGwin G, Apte M, et al. Adolescent onset of lupus results in more aggressive disease and worse outcomes: results of a nested matched case-control study within LUMINA, a multiethnic US cohort (LUMINA LVII). Lupus. 2008;17(4):314-22.

6. Brunner HI, Gladman DD, Ibanez D, Urowitz MD, Silverman ED. Difference in disease features between childhood-onset and adult-onset systemic lupus erythematosus. Arthritis Rheum. 2008;58(2):556-62.

7. Hersh AO, Trupin L, Yazdany J, Panopalis P, Julian L, Katz P, et al. Childhoodonset disease as a predictor of mortality in an adult cohort of patients with systemic lupus erythematosus. Arthritis Care Res (Hoboken). 2010;62(8): 1152-9.

8. Quinlan C, Marks SD, Tullus K. Why are kids with lupus at an increased risk of cardiovascular disease? Pediatr Nephrol. 2016;31(6):861-83.

9. Hersh $A O$, von Scheven E, Yazdany J, Panopalis P, Trupin L, Julian L, et al. Differences in long-term disease activity and treatment of adult patients with childhood- and adult-onset systemic lupus erythematosus. Arthritis Rheum. 2009:61(1):13-20.

10. Esdaile JM, Abrahamowicz M, Grodzicky T, Li Y, Panaritis C, du Berger R, et al. Traditional Framingham risk factors fail to fully account for accelerated atherosclerosis in systemic lupus erythematosus. Arthritis Rheum. 2001; 44(10):2331-7.

11. de Ferranti SD, Steinberger J, Ameduri R, Baker A, Gooding H, Kelly AS, et al. Cardiovascular Risk Reduction in High-Risk Pediatric Patients: A Scientific Statement From the American Heart Association. Circulation. 2019;139(13): e603-e34.

12. Expert Panel on Integrated Guidelines for Cardiovascular. H, Risk Reduction in C, Adolescents, National Heart, Blood L I. Expert panel on integrated 
guidelines for cardiovascular health and risk reduction in children and adolescents: summary report. Pediatrics. 2011;128 Suppl 5:S213-56.

13. Costenbader KH, Wright E, Liang MH, Karlson EW. Cardiac risk factor awareness and management in patients with systemic lupus erythematosus. Arthritis Rheum. 2004;51(6):983-8.

14. Leung YY, Yao Hui LL, Kraus VB. Colchicine-Update on mechanisms of action and therapeutic uses. Semin Arthritis Rheum. 2015;45(3):341-50.

15. Tardif JC, Kouz S, Waters DD, Bertrand OF, Diaz R, Maggioni AP, et al. Efficacy and Safety of Low-Dose Colchicine after Myocardial Infarction. N Engl J Med. 2019;381(26):2497-505.

16. Zinger $\mathrm{H}$, Sherer $\mathrm{Y}$, Shoenfeld $\mathrm{Y}$. Atherosclerosis in autoimmune rheumatic diseases-mechanisms and clinical findings. Clin Rev Allergy Immunol. 2009; 37(1):20-8.

17. Hansson GK, Robertson AK, Soderberg-Naucler C. Inflammation and atherosclerosis. Annu Rev Pathol. 2006;1:297-329.

18. Sherer $Y$, Shoenfeld Y. Mechanisms of disease: atherosclerosis in autoimmune diseases. Nat Clin Pract Rheumatol. 2006;2(2):99-106.

19. Bhatia GS, Sosin MD, Khattak FH, Davis RC, Lip GY. Rheumatoid disease and ischaemic heart disease: insights from pathophysiology and vascular biology. Int J Cardiol. 2005;105(1):1-10.

20. Dinarello CA. Interleukin-1 in the pathogenesis and treatment of inflammatory diseases. Blood. 2011;117(14):3720-32.

21. Dinarello CA, Simon A, van der Meer JW. Treating inflammation by blocking interleukin-1 in a broad spectrum of diseases. Nat Rev Drug Discov. 2012; 11(8):633-52.

22. Furman D, Chang J, Lartigue L, Bolen CR, Haddad F, Gaudilliere B, et al. Expression of specific inflammasome gene modules stratifies older individuals into two extreme clinical and immunological states. Nat Med. 2017:23(2):174-84.

23. Kirii H, Niwa T, Yamada Y, Wada H, Saito K, Iwakura Y, et al. Lack of interleukin-1 beta decreases the severity of atherosclerosis in ApoE-deficient mice. Arterioscler Thromb Vasc Biol. 2003;23(4):656-60.

24. Shimokawa H, Ito A, Fukumoto Y, Kadokami T, Nakaike R, Sakata M, et al. Chronic treatment with interleukin-1 beta induces coronary intimal lesions and vasospastic responses in pigs in vivo. The role of platelet-derived growth factor. J Clin Invest. 1996;97(3):769-76.

25. Thompson PL, Nidorf SM. Anti-inflammatory therapy with canakinumab for atherosclerotic disease: lessons from the CANTOS trial. J Thorac Dis. 2018; 10(2):695-8

26. McGill HC Jr, McMahan CA. Determinants of atherosclerosis in the young. Pathobiological Determinants of Atherosclerosis in Youth (PDAY) Research Group. Am J Cardiol. 1998;82(10B):30T-6T.

27. Hayata AL, Borba EF, Bonfa E, Kochen JA, Goldenstein-Schainberg C. The frequency of high/moderate lipoprotein risk factor for coronary artery disease is significant in juvenile-onset systemic lupus erythematosus. Lupus. 2005;14(8):613-7.

28. Tyrrell PN, Beyene J, Benseler SM, Sarkissian T, Silverman ED. Predictors of lipid abnormalities in children with new-onset systemic lupus erythematosus. J Rheumatol. 2007;34(10):2112-9.

29. Carroll MD, Kit BK, Lacher DA, Shero ST, Mussolino ME. Trends in lipids and lipoproteins in US adults, 1988-2010. JAMA. 2012;308(15):1545-54.

30. Friedman DM, Lazarus HM, Fierman $\mathrm{AH}$. Acute myocardial infarction in pediatric systemic lupus erythematosus. J Pediatr. 1990;117(2 Pt 1): 263-6.

31. Korkmaz C, Cansu DU, Kasifoglu T. Myocardial infarction in young patients ( $<$ or $=35$ years of age) with systemic lupus erythematosus: a case report and clinical analysis of the literature. Lupus. 2007;16(4):289-97.

32. Chang JC, Xiao R, Mercer-Rosa L, Knight AM, Weiss PF. Child-onset systemic lupus erythematosus is associated with a higher incidence of myopericardial manifestations compared to adult-onset disease. Lupus. 2018:27(13):2146-54

33. Roldan CA, Shively BK, Crawford MH. An echocardiographic study of valvular heart disease associated with systemic lupus erythematosus. N Engl J Med. 1996;335(19):1424-30.

34. Wijetunga M, Rockson S. Myocarditis in systemic lupus erythematosus. Am J Med. 2002;113(5):419-23.

35. Schanberg LE, Sandborg C, Barnhart HX, Ardoin SP, Yow E, Evans GW, et al. Premature atherosclerosis in pediatric systemic lupus erythematosus: risk factors for increased carotid intima-media thickness in the atherosclerosis prevention in pediatric lupus erythematosus cohort. Arthritis Rheum. 2009; 60(5):1496-507.
36. Haque S, Gordon C, Isenberg D, Rahman A, Lanyon P, Bell A, et al. Risk factors for clinical coronary heart disease in systemic lupus erythematosus: the lupus and atherosclerosis evaluation of risk (LASER) study. J Rheumatol. 2010;37(2):322-9.

37. Bessant R, Duncan R, Ambler G, Swanton J, Isenberg DA, Gordon C, et al. Prevalence of conventional and lupus-specific risk factors for cardiovascular disease in patients with systemic lupus erythematosus: A case-control study. Arthritis Rheum. 2006;55(6):892-9.

38. Kavanaugh A, Adams-Huet B, Jain R, Denke M, McFarlin J. Hydroxychloroquine Effects on Lipoprotein Profiles (the HELP trial): A Double-Blind, Randomized, Placebo-Controlled, Pilot Study In Patients With Systemic Lupus Erythematosus. J Clin Rheumatol. 1997;3(1):3-8.

39. Tanay A, Leibovitz E, Frayman A, Zimlichman R, Shargorodsky M, Gavish D. Vascular elasticity of systemic lupus erythematosus patients is associated with steroids and hydroxychloroquine treatment. Ann N Y Acad Sci. 2007: 1108:24-34

40. Greenwood J, Steinman L, Zamvil SS. Statin therapy and autoimmune disease: from protein prenylation to immunomodulation. Nat Rev Immunol. 2006:6(5):358-70.

41. Petri MA, Kiani AN, Post W, Christopher-Stine L, Magder LS. Lupus Atherosclerosis Prevention Study (LAPS). Ann Rheum Dis. 2011:70(5):760-5.

42. Schanberg LE, Sandborg C, Barnhart HX, Ardoin SP, Yow E, Evans GW, et al. Use of atorvastatin in systemic lupus erythematosus in children and adolescents. Arthritis Rheum. 2012;64(1):285-96.

43. Ardoin SP, Schanberg LE, Sandborg Cl, Barnhart HX, Evans GW, Yow E, et al. Secondary analysis of APPLE study suggests atorvastatin may reduce atherosclerosis progression in pubertal lupus patients with higher $C$ reactive protein. Ann Rheum Dis. 2014;73(3):557-66.

44. Schlesinger N. Management of acute and chronic gouty arthritis: present state-of-the-art. Drugs. 2004;64(21):2399-416.

45. Ravelli RB, Gigant B, Curmi PA, Jourdain I, Lachkar S, Sobel A, et al. Insight into tubulin regulation from a complex with colchicine and a stathmin-like domain. Nature. 2004;428(6979):198-202.

46. Cronstein BN, Molad Y, Reibman J, Balakhane E, Levin Rl, Weissmann G. Colchicine alters the quantitative and qualitative display of selectins on endothelial cells and neutrophils. J Clin Invest. 1995:96(2):994-1002.

47. Asako H, Kubes P, Baethge BA, Wolf RE, Granger DN. Colchicine and methotrexate reduce leukocyte adherence and emigration in rat mesenteric venules. Inflammation. 1992;16(1):45-56.

48. Roberge CJ, Gaudry M, de Medicis R, Lussier A, Poubelle PE, Naccache PH. Crystal-induced neutrophil activation. IV. Specific inhibition of tyrosine phosphorylation by colchicine. J Clin Invest. 1993;92(4):1722-9.

49. Chae JJ, Wood G, Richard K, Jaffe H, Colburn NT, Masters SL, et al. The familial Mediterranean fever protein, pyrin, is cleaved by caspase- 1 and activates NF-kappaB through its N-terminal fragment. Blood. 2008;112(5): 1794-803.

50. Martinon F, Petrilli V, Mayor A, Tardivel A, Tschopp J. Gout-associated uric acid crystals activate the NALP3 inflammasome. Nature. 2006;440(7081):237-41.

51. Libby P. Interleukin-1 Beta as a Target for Atherosclerosis Therapy: Biological Basis of CANTOS and Beyond. J Am Coll Cardiol. 2017;70(18):2278-89.

52. Loppnow H, Libby P. Proliferating or interleukin 1-activated human vascular smooth muscle cells secrete copious interleukin 6. J Clin Invest. 1990;85(3): 731-8.

53. Bevilacqua MP, Pober JS, Wheeler ME, Cotran RS, Gimbrone MA Jr. Interleukin-1 activation of vascular endothelium. Effects on procoagulant activity and leukocyte adhesion. Am J Pathol. 1985;121(3):394-403.

54. Terekeci HM, Oktenli C, Ozgurtas T, Nalbant S, Top C, Celik S, et al. Increased asymmetric dimethylarginine levels in young men with familial Mediterranean fever (FMF): is it early evidence of interaction between inflammation and endothelial dysfunction in FMF? J Rheumatol. 2008;35(10): 2024-9.

55. Atta HM, El-Rehany MA, Abdel Raheim SR, Fouad R, Galal AM. Colchicine inhibits intimal hyperplasia and leukocyte VEGF expression in dogs. I Surg Res. 2008:146(2):184-9.

56. Lee FY, Lu HI, Zhen YY, Leu S, Chen YL, Tsai TH, et al. Benefit of combined therapy with nicorandil and colchicine in preventing monocrotalineinduced rat pulmonary arterial hypertension. Eur J Pharm Sci. 2013;50(3-4) 372-84.

57. Huang C, Cen C, Wang C, Zhan H, Ding X. Synergistic effects of colchicine combined with atorvastatin in rats with hyperlipidemia. Lipids Health Dis. 2014;13:67. 
58. Morel N, Bonjour M, Le Guern V, Le Jeunne C, Mouthon L, Piette JC, et al. Colchicine: a simple and effective treatment for pericarditis in systemic lupus erythematosus? A report of 10 cases. Lupus. 2015;24(14):1479-85.

59. Padeh $\mathrm{S}$, Gerstein M, Berkun Y. Colchicine is a safe drug in children with familial Mediterranean fever. J Pediatr. 2012;161(6):1142-6.

60. Kallinich T, Haffner D, Niehues T, Huss K, Lainka E, Neudorf U, et al. Colchicine use in children and adolescents with familial Mediterranean fever: literature review and consensus statement. Pediatrics. 2007;119(2): e474-83.

61. Zemer D, Livneh A, Danon YL, Pras M, Sohar E. Long-term colchicine treatment in children with familial Mediterranean fever. Arthritis Rheum. 1991;34(8):973-7.

62. Tateishi T, Soucek P, Caraco Y, Guengerich FP, Wood AJ. Colchicine biotransformation by human liver microsomes. Identification of CYP3A4 as the major isoform responsible for colchicine demethylation. Biochem Pharmacol. 1997;53(1):111-6.

63. Nidorf SM, Eikelboom JW, Budgeon CA, Thompson PL. Low-dose colchicine for secondary prevention of cardiovascular disease. J Am Coll Cardiol. 2013; 61(4):404-10.

64. Lopez-Melgar B, Fernandez-Friera L, Oliva B, Garcia-Ruiz JM, Sanchez-Cabo F, Bueno $\mathrm{H}$, et al. Short-Term Progression of Multiterritorial Subclinical Atherosclerosis. J Am Coll Cardiol. 2020;75(14):1617-27.

65. Kaptoge S, Seshasai SR, Gao P, Freitag DF, Butterworth AS, Borglykke A, et al. Inflammatory cytokines and risk of coronary heart disease: new prospective study and updated meta-analysis. Eur Heart J. 2014;35(9):578-89.

66. Daiber A, Xia N, Steven S, Oelze M, Hanf A, Kroller-Schon S, et al. New Therapeutic Implications of Endothelial Nitric Oxide Synthase (eNOS) Function/Dysfunction in Cardiovascular Disease. Int J Mol Sci. 2019;20(1).

\section{Publisher's Note}

Springer Nature remains neutral with regard to jurisdictional claims in published maps and institutional affiliations.

Ready to submit your research? Choose BMC and benefit from:

- fast, convenient online submission

- thorough peer review by experienced researchers in your field

- rapid publication on acceptance

- support for research data, including large and complex data types

- gold Open Access which fosters wider collaboration and increased citations

- maximum visibility for your research: over $100 \mathrm{M}$ website views per year

At $\mathrm{BMC}$, research is always in progress.

Learn more biomedcentral.com/submissions 